\title{
A Note on Nearly Sasakian and Nearly Cosymplictic Structures of 5-Dimensional Spheres
}

\author{
Sharief Deshmukh* and Ibrahim Al-Dayel
}

(Communicated by Kazım İlarslan)

\begin{abstract}
In this paper, we show that with the nearly Sasakian structure $(\varphi, \xi, \eta, g)$ on the 5 -dimensional sphere $S^{5}(2)$ of constant curvature 2 (cf. [2]), there are naturally associated two additional structures $\left(\varphi_{1}, \xi, \eta, g\right),\left(\varphi_{2}, \xi, \eta, g\right)$ on $S^{5}(2)$, where $S^{5}(2)\left(\varphi_{1}, \xi, \eta, g\right)$ is homothetic to a Sasakian manifold and $S^{5}(2)\left(\varphi_{2}, \xi, \eta, g\right)$ is a nearly cosymplectic manifold. Similarly, we show that on the unit sphere $S^{5}$, which is known to have a nearly cosymplectic structure $\left(\psi_{1}, \xi, \eta, g\right)$ (cf. [2]), there are two additional structures $\left(\psi_{2}, \xi, \eta, g\right),\left(\psi_{3}, \xi, \eta, g\right)$ on $S^{5}$ such that $S^{5}\left(\psi_{2}, \xi, \eta, g\right)$ is a Sasakian manifold and $S^{5}\left(\psi_{3}, \xi, \eta, g\right)$ is a nearly cosymplectic manifold and the last nearly cosymplectic structure is independent of the nearly cosymplectic structure $\left(\psi_{1}, \xi, \eta, g\right)$, in the sense that these three structures satisfy $\psi_{1} \psi_{2}=-\psi_{2} \psi_{1}=\psi_{3}, \psi_{2} \psi_{3}=-\psi_{3} \psi_{2}=\psi_{1}$ and $\psi_{3} \psi_{1}=-\psi_{1} \psi_{3}=\psi_{2}$.
\end{abstract}

Keywords: Nearly Kaehler manifolds; Nearly Sasakian manifolds; Nearly cosymplectic manifolds; Sasakian manifolds.

AMS Subject Classification (2010): Primary: 53B25; Secondary: 53C25.

\section{Introduction}

Recall that, as an odd dimensional analogue of the nearly Kaehler structure on the unit sphere $S^{6}$, which is not Kaehler [4], in [2,3], nearly Sasakian structure was introduced on the 5-dimensional sphere $S^{5}(2)$ of constant curvature 2 as totally umbilical hypersurface of $S^{6}$, which is not a Sasakian structure. However, the nearly Kaehler structure on $S^{6}$ is not related to any Kaehler structure as there are no known Kaehler structures on the six dimensional sphere $S^{6}$. Though, in case of the 5-dimensional sphere $S^{5}(2)$, which is a nearly Sasakian manifold, it does not admit any Sasakian structure owing to the fact that $S^{5}(2)$ has constant sectional curvature 2 and a Sasakian structure requires sectional curvatures of the plane sections containing Reeb vector field $\xi$ to be the constant 1 . However, in this paper, it is shown that the nearly structure $(\varphi, \xi, \eta, g)$ on $S^{5}(2)$ naturally induces two more structures $\left(\varphi_{1}, \xi, \eta, g\right),\left(\varphi_{2}, \xi, \eta, g\right)$ on $S^{5}(2)$ such that $S^{5}(2)\left(\varphi_{1}, \xi, \eta, g\right)$ is homothetic to a Sasakian manifold and $S^{5}(2)\left(\varphi_{1}, \xi, \eta, g\right)$ is a nearly cosymplectic manifold. Thus the link that nearly nearly Kaehler structure of $S^{6}$ is not related to any Kaehler structure is broken in case of the nearly Sasakian structure of $S^{5}(2)$.

Similarly, it is known that the unit sphere $S^{5}$ as totally geodesic hypersurface of the nearly Kaehler 6 -sphere $S^{6}$ inherits a nearly cosymplectic structure $\left(\psi_{1}, \xi, \eta, g\right)$ [2], which is not cosymplectic. In this paper, we show that there are two additional structures $\left(\psi_{i}, \xi, \eta, g\right), i=2,3$ associated to the nearly cosymplectic structure $\left(\psi_{1}, \xi, \eta, g\right)$ on $S^{5}$ of which $\left(\psi_{2}, \xi, \eta, g\right)$ is Sasakian and $\left(\psi_{3}, \xi, \eta, g\right)$ is nearly cosymplectic structure and these structures satisfy

$$
\psi_{1} \psi_{2}=-\psi_{2} \psi_{1}=\psi_{3}, \quad \psi_{2} \psi_{3}=-\psi_{3} \psi_{2}=\psi_{1}, \quad \psi_{3} \psi_{1}=-\psi_{1} \psi_{3}=\psi_{2},
$$

that is, the new nearly cosymplectic structure $\left(\psi_{3}, \xi, \eta, g\right)$ on $S^{5}$ is independent of the original nearly cosymplectic structure $\left(\psi_{1}, \xi, \eta, g\right)$ of $S^{5}$.

Received : 01-02-2018, Accepted : 18-06-2018

* Corresponding author 


\section{Preliminaries}

Let $M$ be a real hypersurface of the nearly Kaehler 6 -sphere $\left(S^{6}, J, \bar{g}\right)$ with unit normal vector field $N$. Then the hypersurface $M$ admits an almost contact metric structure $(\varphi, \xi, \eta, g)$, where $g$ is the induced metric and

$$
J X=\varphi X+\eta(X) N, \quad J \xi=N, \quad X, Y \in \mathfrak{X}(M),
$$

where $\eta$ is the smooth 1 -form dual to the unit vector field $\xi, \varphi X$ is the tangential component of $J X$ and $\mathfrak{X}(M)$ is the Lie algebra of smooth vector fields on the hypersurface $M$. The almost contact metric structure $(\varphi, \xi, \eta, g)$ satisfies

$$
\varphi^{2}=-I+\eta \otimes \xi, \varphi(\xi)=0, \eta \circ \varphi=0,
$$

and

$$
g(\varphi X, \varphi Y)=g(X, Y)-\eta(X) \eta(Y) .
$$

In [3], it is shown that the 5-dimensional sphere $S^{5}(2)$ of constant curvature 2 as totally umbilical hypersurface of the nearly Kaehler 6 -sphere $\left(S^{6}, J, \bar{g}\right)$ is a nearly nearly Sasakian manifold, that is, it admits an almost contact metric structure $(\varphi, \xi, \eta, g)$ that satisfies

$$
(\nabla \varphi)(X, Y)+(\nabla \varphi)(Y, X)=\eta(Y) X+\eta(X) Y-2 g(X, Y) \xi, \quad X \in \mathfrak{X}\left(S^{5}(2)\right),
$$

where $(\nabla \varphi)(X, Y)=\nabla_{X} \varphi Y-\varphi\left(\nabla_{X} Y\right), \nabla$ is covariant derivative operator with respect to the induced metric $g$ (see also [2], [6], [7]).

Also, in ([2], [3]), it is shown that the unit 5-sphere $S^{5}$ as totally geodesic hypersurface of the nearly Kaehler 6sphere $\left(S^{6}, J, \bar{g}\right)$ is a nearly cosymplectic manifold, that is, it admits an almost contact metric structure $(\varphi, \xi, \eta, g)$ that satisfies

$$
(\nabla \varphi)(X, Y)+(\nabla \varphi)(Y, X)=0, \quad X \in \mathfrak{X}\left(S^{5}\right) .
$$

An almost contact metric manifold $M(\varphi, \xi, \eta, g)$ is said to be a Sasakian manifold if the following holds

$$
(\nabla \varphi)(X, Y)=\eta(Y) X-g(X, Y) \xi, \quad X \in \mathfrak{X}(M) .
$$

It follows from above equation that a Sasakian manifold is a nearly Sasakian manifold but the converse is not true. In particular, the nearly Sasakian manifold $S^{5}(2)(\varphi, \xi, \eta, g)$ is not a Sasakian manifold as a Sasakian manifold requires sectional curvatures of the plane sections containing the Reeb vector field $\xi$ to be constant 1 .

A smooth vector field $\xi$ on a Riemannian manifold $(M, g)$ is said to be a Killing vector field if its flow consists of isometries of the Riemannian manifold $(M, g)$ or equivalently

$$
£_{\xi} g=0,
$$

where $£_{\xi}$ is the Lie derivative with respect to $\xi$. If $\eta$ is a smooth 1-form dual to the Killing vector field $\xi$ and if we define a skew-symmetric $(1,1)$ tensor field $\psi$ on $M$ by $d \eta(X, Y)=2 g(\psi X, Y), X, Y \in \mathfrak{X}(M)$, then using Koszul's formula (cf [1]), we get

$$
\nabla_{X} \xi=\psi X, \quad X \in \mathfrak{X}(M) .
$$

The above equation, immediately gives the following expression

$$
R(X, Y) \xi=(\nabla \psi)(X, Y)-(\nabla \psi)(Y, X), \quad X, Y \in \mathfrak{X}(M),
$$

where $R$ is the curvature tensor of the Riemannian manifold $(M, g)$. Also, as the smooth 2 -form $\Omega(X, Y)=$ $g(\psi X, Y)$ is closed, we have

$$
g((\nabla \psi)(X, Y), Z)+g((\nabla \psi)(Y, Z), X)+g((\nabla \psi)(Z, X), Y)=0,
$$

which together with skew-symmetry of $\psi$ and the equation (2.5), gives

$$
(\nabla \psi)(X, Y)=R(X, \xi) Y, \quad X, Y \in \mathfrak{X}(M) .
$$




\section{Nearly Sasakian manifold $S^{5}(2)(\varphi, \xi, \eta, g)$}

In this section, we investigate the existence of other structures on the nearly Sasakian manifold $S^{5}(2)(\varphi, \xi, \eta, g)$. Recall that the Reeb vector field $\xi$ on the nearly Sasakian manifold $S^{5}(2)(\varphi, \xi, \eta, g)$, is Killing $[2,3]$ and hence there exists a skew-symmetric tensor field $\varphi_{1}$ on $S^{5}(2)$ satisfying

$$
\nabla_{X} \xi=\varphi_{1} X, \quad X \in \mathfrak{X}\left(S^{5}(2)\right)
$$

and by equation (2.6), we have

$$
\left(\nabla \varphi_{1}\right)(X, Y)=R(X, \xi) Y=2(g(Y, \xi) X-g(X, Y) \xi), \quad X, Y \in \mathfrak{X}\left(S^{5}(2)\right) .
$$

Taking $Y=\xi$, we have

$$
\varphi_{1}^{2} X=2(-X+\eta(X) \xi),
$$

and as $\xi$ is a unit vector field, the equation (3.1) gives $\varphi_{1}(\xi)=\nabla_{\xi} \xi=0$. Moreover, equations (3.1) and (3.2), give

$$
\nabla_{X} \nabla_{Y} \xi-\nabla_{\nabla_{X} Y} \xi=\left(\nabla \varphi_{1}\right)(X, Y)=2(g(Y, \xi) X-g(X, Y) \xi),
$$

which by a result in [5], implies that $S^{5}(2)\left(\varphi_{1}, \xi, \eta, g\right)$ is homothetic to a Sasakian manifold. Indeed, this new Sasakian structure $\left(\varphi_{1}^{\prime}, \xi^{\prime}, \eta^{\prime}, g^{\prime}\right)$ on $S^{5}(2)$ is given by

$$
\varphi_{1}=\sqrt{2} \varphi_{1}^{\prime}, \xi=\sqrt{2} \xi^{\prime}, \eta=\frac{1}{\sqrt{2}} \eta^{\prime}, g=\frac{1}{2} g^{\prime} .
$$

Now, define a $(1,1)$ tensor field $\varphi_{2}$ on the nearly Sasakian manifold $S^{5}(2)(\varphi, \xi, \eta, g)$ by

$$
\varphi_{2}(X)=(\nabla \varphi)(\xi, X), \quad X \in \mathfrak{X}\left(S^{5}(2)\right),
$$

then by equation (2.1), it follows that $\varphi_{2}(\xi)=0$ and that $\varphi_{2}$ is a skew-symmetric tensor. First, we investigate the relations between these three operators $\varphi_{,} \varphi_{1}$ and $\varphi_{2}$ in the following:

Lemma 3.1. The operators $\varphi_{1}$ and $\varphi_{2}$ on the nearly Sasakian manifold $S^{5}(2)(\varphi, \xi, \eta, g)$ satisfy

$$
\begin{aligned}
\varphi_{1} X & \left.=\varphi \varphi_{1} \varphi X+2 \varphi X, \quad \frac{1}{2}\left(\varphi\left(\varphi_{1} X\right)+\varphi_{1}(\varphi X)\right)\right)=-X+\eta(X) \xi, \\
\varphi_{2} X & =\frac{1}{2}\left(\varphi \varphi_{1} X-\varphi_{1} \varphi X\right), \quad X, Y \in X\left(S^{5}(2)\right) .
\end{aligned}
$$

Proof. Taking $Y=\xi$ in equation (2.1), we get

$$
-\varphi\left(\nabla_{X} \xi\right)+(\nabla \varphi)(X, Y)=X-\eta(X) \xi,
$$

which gives,

$$
\varphi_{1} X=\varphi X-\varphi \varphi_{2} X,
$$

that is,

$$
\varphi \varphi_{1} X=-X+\eta(X) \xi+\varphi_{2} X,
$$

where we used $\varphi_{2}(\xi)=0$. Now, replacing $X$ by $\varphi X$ in (3.4), we conclude

$$
\varphi_{1} \varphi X=-X+\eta(X) \xi-\varphi \varphi_{2} \varphi X .
$$

We have

$$
\begin{aligned}
\varphi \varphi_{2} \varphi X & =\varphi(\nabla \varphi)(\xi, \varphi X)=\varphi\left[-\nabla_{\xi} X+\eta\left(\nabla_{\xi} X\right) \xi-\varphi\left(\nabla_{\xi} \varphi X\right)\right] \\
& =\varphi\left[-\nabla_{\xi} X-\varphi\left((\nabla \varphi)(\xi, X)+\varphi\left(\nabla_{\xi} X\right)\right)\right] \\
& =\varphi\left[-\nabla_{\xi} X-\varphi \varphi_{2} X+-\nabla_{\xi} X+\eta\left(\nabla_{\xi} X\right) \xi\right] \\
& =\varphi_{2} X
\end{aligned}
$$


consequently, equation (3.6) takes the form

$$
\varphi_{1} \varphi X=-X+\eta(X) \xi-\varphi_{2} X .
$$

Then equations (3.5) and (3.7), give

$$
\left.\left.\left.\varphi_{2} X=\frac{1}{2}\left(\varphi \varphi_{1} X\right)-\varphi_{1} \varphi X\right)\right) \text { and } \frac{1}{2}\left(\varphi\left(\varphi_{1} X\right)+\varphi_{1}(\varphi X)\right)\right)=-X+\eta(X) \xi,
$$

which on substituting $\varphi_{2} X$ in equation (3.4) proves the Lemma.

Now, we are in position to prove the following:

Theorem 3.1. There are two structures $\left(\varphi_{i}, \xi, \eta, g\right), i=1,2$ associated to the nearly Sasakian structure $(\varphi, \xi, \eta, g)$ on the 5 -sphere $S^{5}(2)$ such that $S^{5}(2)\left(\varphi_{1}, \xi, \eta, g\right)$ is homothetic to a Sasakian manifold and $S^{5}(2)\left(\varphi_{2}, \xi, \eta, g\right)$ is a nearly cosymplectic manifold.

Proof. It remains to prove that $\left(\varphi_{2}, \xi, \eta, g\right)$ is a nearly cosymplectic structure on $S^{5}(2)$. We use equation (2.1), to compute

$$
\begin{aligned}
\varphi_{2}^{2} X & =(\nabla \varphi)(\xi,(\nabla \varphi)(\xi, X)) \\
& =-(\nabla \varphi)((\nabla \varphi)(\xi, X), \xi)+(\nabla \varphi)(\xi, X),
\end{aligned}
$$

where we used $\eta((\nabla \varphi)(\xi, X))=g((\nabla \varphi)(\xi, X), \xi)=-g(X,(\nabla \varphi)(\xi, \xi))=0$. Thus, again on using equation $(2.1)$,

$$
\begin{aligned}
\varphi_{2}^{2} X & =-(\nabla \varphi)(-(\nabla \varphi)(X, \xi)-\eta(X) \xi+X, \xi)-(\nabla \varphi)(X, \xi)-\eta(X) \xi+X \\
& \left.=-(\nabla \varphi) \varphi\left(\nabla_{X} \xi\right), \xi\right)-2(\nabla \varphi)(X, \xi)-\eta(X) \xi+X \\
& =\varphi\left(\nabla_{\varphi\left(\nabla_{X} \xi\right)} \xi\right)+2 \varphi\left(\nabla_{X} \xi\right)-\eta(X) \xi+X \\
& =\varphi \varphi_{1} \varphi \varphi_{1} X+2 \varphi \varphi_{1} X-\eta(X) \xi+X \\
& =\left(\varphi \varphi_{1} \varphi+2 \varphi\right) \varphi_{1} X-\eta(X) \xi+X
\end{aligned}
$$

which by Lemma 3.1, gives

$$
\varphi_{2}^{2} X=\varphi_{1}^{2} X-\eta(X) \xi+X=-X+\eta(X) \xi
$$

where we used equation (3.3). Also, as $\varphi_{2}$ is skew symmetric, we have

$$
g\left(\varphi_{2} X, \varphi_{2} Y\right)=-g\left(\varphi_{2}^{2} X, Y\right)=g(X, Y)-\eta(X) \eta(Y) .
$$

Hence, $\left(\varphi_{2}, \xi, \eta, g\right)$ is an almost contact metric structure on $S^{5}(2)$. Finally, on using equations (2.1), (3.1) and Lemma 2.1, we have

$$
\begin{aligned}
\left(\nabla \varphi_{2}\right)(X, Y)= & \nabla_{X}(\nabla \varphi)(\xi, Y)-(\nabla \varphi)\left(\xi, \nabla_{X} Y\right) \\
= & \nabla_{X}(-(\nabla \varphi)(Y, \xi)-\eta(Y) \xi+Y)+(\nabla \varphi)\left(\nabla_{X} Y, \xi\right)+\eta\left(\nabla_{X} Y\right) \xi-\nabla_{X} Y \\
= & \nabla_{X}\left(\varphi \varphi_{1} Y\right)-X(\eta(Y)) \xi-\eta(Y) \varphi_{1} X-\varphi \varphi_{1}\left(\nabla_{X} Y\right)+\eta\left(\nabla_{X} Y\right) \xi \\
= & \nabla_{X}\left(-\varphi_{1} \varphi Y-2 Y+2 \eta(Y) \xi\right)-X(\eta(Y)) \xi-\eta(Y) \varphi_{1} X+\varphi_{1} \varphi\left(\nabla_{X} Y\right) \\
& +2 \nabla_{X} Y-2 \eta\left(\nabla_{X} Y\right) \xi+\eta\left(\nabla_{X} Y\right) \xi \\
= & -\left[\left(\nabla \varphi_{1}\right)(X, \varphi Y)+\varphi_{1}(\nabla \varphi)(X, Y)+\varphi_{1} \varphi \nabla_{X} Y\right]+X(\eta(Y)) \xi \\
& +\eta(Y) \varphi_{1} X-\eta\left(\nabla_{X} Y\right) \xi+\varphi_{1} \varphi\left(\nabla_{X} Y\right) \\
= & -\left(\nabla \varphi_{1}\right)(X, \varphi Y)-\varphi_{1}(\nabla \varphi)(X, Y)+g\left(Y, \varphi_{1} X\right) \xi+\eta(Y) \varphi_{1} X .
\end{aligned}
$$

Now, using equation (3.2), we conclude

$$
\left(\nabla \varphi_{2}\right)(X, Y)=2 g(X, \varphi Y) \xi-\varphi_{1}(\nabla \varphi)(X, Y)+g\left(Y, \varphi_{1} X\right) \xi+\eta(Y) \varphi_{1} X,
$$

which in view of equation (2.1), gives

$$
\begin{aligned}
\left(\nabla \varphi_{2}\right)(X, Y)+\left(\nabla \varphi_{2}\right)(Y, X)= & -\varphi_{1}(\eta(Y) X+\eta(X) Y-2 g(X, Y) \xi) \\
& +\eta(Y) \varphi_{1} X++\eta(X) \varphi_{1} Y \\
= & 0 .
\end{aligned}
$$

Hence, $\left(\varphi_{2}, \xi, \eta, g\right)$ is a nearly cosymplectic structure on $S^{5}(2)$. 


\section{Nearly cosymplictic manifold $S^{5}\left(\psi_{1}, \xi, \eta, g\right)$}

Recall that the totally geodesic hypersphere $S^{5}$ of the nearly Kaehler 6 -sphere $\left(S^{6}, J, \bar{g}\right)$ admits an almost contact metric structure $\left(\psi_{1}, \xi, \eta, g\right)$ satisfying $J X=\psi_{1} X+\eta(X) N, X \in \mathfrak{X}\left(S^{5}\right)$, where $\psi_{1} X$ is the tangential component of $J X$ and $N$ is the unit normal vector field to $S^{5}$, which is related to the Reeb vector field $\xi$ by $J \xi=N$ and $\eta$ is smooth 1 -form dual to the unit vector field $\xi$. It follows that the almost contact metric structure $\left(\psi_{1}, \xi, \eta, g\right)$ on $S^{5}$ is nearly cosymplectic, that is, it satisfies

$$
\left(\nabla \psi_{1}\right)(X, Y)+\left(\nabla \psi_{1}\right)(Y, X)=0, \quad X, Y \in \mathfrak{X}\left(S^{5}\right),
$$

where $\left(\nabla \psi_{1}\right)(X, Y)=\nabla_{X} \psi_{1} Y-\psi_{1}\left(\nabla_{X} Y\right)$ (cf. [2], [3]). Also, it is known that the Reeb vector field $\xi$ on the nearly cosymplectic manifold $S^{5}\left(\psi_{1}, \xi, \eta, g\right)$ is Killing (cf. [2]) and consequently, there is a $(1,1)$ skewsymmetric tensor field $\psi_{2}$ on the nearly cosymplectic manifold $S^{5}\left(\psi_{1}, \xi, \eta, g\right)$ defined by $d \eta(X, Y)=2 g\left(\psi_{2} X, Y\right)$, $X, Y \in \mathfrak{X}\left(S^{5}\right)$, which by Koszul's formula satisfies

$$
\nabla_{X} \xi=\psi_{2} X, \quad X \in \mathfrak{X}\left(S^{5}\right)
$$

and using the fact that the smooth 2-form $\Omega_{2}(X, Y)=g\left(\psi_{2} X, Y\right)$ is closed, we arrive at

$$
\left(\nabla \psi_{2}\right)(X, Y)=R(X, \xi) Y=\eta(Y) X-g(X, Y) \xi .
$$

Since, $\xi$ is a unit Killing vector field and $\psi_{2}$ is skew-symmetric, equation (4.2) gives $\psi_{2}(\xi)=\nabla_{\xi} \xi=0$. Taking $Y=\xi$ in equation (4.3) and using $\psi_{2}(\xi)=0$, we get

$$
\psi_{2}^{2}(X)=-X+\eta(X) \xi, \quad X \in \mathfrak{X}\left(S^{5}\right),
$$

and it follows that $g\left(\psi_{1}(X), \psi_{2}(Y)\right)=g(X, Y)-\eta(X) \eta(Y)$. Hence, $\left(\psi_{2}, \xi, \eta, g\right)$ is an almost contact metric structure on $S^{5}\left(\psi_{1}, \xi, \eta, g\right)$ and equation (4.3) confirms that $\left(\psi_{2}, \xi, \eta, g\right)$ is a Sasakian structure.

Now, define an operator $\psi_{3}$ on the nearly cosymplectic manifold $S^{5}\left(\psi_{1}, \xi, \eta, g\right)$ by

$$
\psi_{3}(X)=\left(\nabla \psi_{1}\right)(\xi, X), \quad X \in \mathfrak{X}\left(S^{5}\right),
$$

which in view of equation (4.1) confirms that $\psi_{3}$ is a $(1,1)$ skew-symmetric tensor field and it satisfies $\psi_{3}(\xi)=0$. Also, equations (4.1) and (4.2), imply that

$$
\psi_{3}(X)=-\left(\nabla \psi_{1}\right)(X, \xi)=\psi_{1}\left(\nabla_{X} \xi\right)=\psi_{1} \psi_{2}(X), \quad X \in \mathfrak{X}\left(S^{5}\right),
$$

which gives

$$
\psi_{2}(X)=-\psi_{1} \psi_{3}(X), \quad X \in \mathfrak{X}\left(S^{5}\right) .
$$

Replacing $X$ by $\psi_{1} X$ in equation (4.5), we get

$$
\psi_{2} \psi_{1}(X)=-\psi_{1} \psi_{3} \psi_{1}(X), \quad X \in \mathfrak{X}\left(S^{5}\right) .
$$

Also, we have

$$
\begin{aligned}
\psi_{1} \psi_{3} \psi_{1}(X) & =\psi_{1}\left[\left(\nabla \psi_{1}\right)\left(\xi, \psi_{1} X\right)\right]=\psi_{1}\left[\nabla_{\xi}(-X+\eta(X) \xi)-\psi_{1}\left(\nabla_{\xi} \psi_{1} X\right)\right] \\
& =\psi_{1}\left[-\nabla_{\xi} X+\xi(\eta(X)) \xi-\psi_{1}\left(\left(\nabla \psi_{1}\right)(\xi, X)+\psi_{1}\left(\nabla_{\xi} X\right)\right)\right] \\
& =\psi_{1}\left[-\nabla_{\xi} X+\xi(\eta(X)) \xi-\psi_{1} \psi_{3} X+\nabla_{\xi} X\right]=\psi_{3} X
\end{aligned}
$$

which in view of equation (4.6) gives

$$
\psi_{2} \psi_{1}(X)=-\psi_{3} X, \quad X \in \mathfrak{X}\left(S^{5}\right) .
$$

Thus, equations (4.4) and (4.7) lead to

$$
\psi_{3}=\psi_{1} \psi_{2}=-\psi_{2} \psi_{1} .
$$

Now, using above equation, we get

$$
\psi_{3}^{2} X=\psi_{1} \psi_{2} \psi_{1} \psi_{2}(X)=-\psi_{1} \psi_{2}^{2} \psi_{1}(X)=\psi_{1}^{2}(X)=-X+\eta(X) \xi,
$$


and

$$
g\left(\psi_{3} X, \psi_{3} Y\right)=g(X, Y)-\eta(X) \eta(Y) .
$$

Hence, $\left(\psi_{3}, \xi, \eta, g\right)$ is an almost contact metric structure on $S^{5}\left(\psi_{1}, \xi, \eta, g\right)$ and the three almost contact structures $\left(\psi_{i}, \xi, \eta, g\right), i=1,2,3$ satisfy equations (4.5)-(4.8), consequently, we have the following

$$
\psi_{1} \psi_{2}=-\psi_{2} \psi_{1}=\psi_{3}, \quad \psi_{2} \psi_{3}=-\psi_{3} \psi_{2}=\psi_{1}, \quad \psi_{3} \psi_{1}=-\psi_{1} \psi_{3}=\psi_{2} .
$$

Thus, we have the following:

Theorem 4.1. There are three $\left(\psi_{i}, \xi, \eta, g\right), i=1,2,3$ almost contact metric structures on the unit sphere $S^{5}$ satisfying

$$
\psi_{1} \psi_{2}=-\psi_{2} \psi_{1}=\psi_{3}, \quad \psi_{2} \psi_{3}=-\psi_{3} \psi_{2}=\psi_{1}, \quad \psi_{3} \psi_{1}=-\psi_{1} \psi_{3}=\psi_{2}
$$

such that $S^{5}\left(\psi_{i}, \xi, \eta, g\right), i=1,3$ are nearly cosymplectic manifolds and $S^{5}\left(\psi_{2}, \xi, \eta, g\right)$ is a Sasakian manifold.

Proof. It remains to prove that the almost contact metric structure $\left(\psi_{3}, \xi, \eta, g\right)$ is a nearly cosymplectic structure on $S^{5}$. We use equations (4.8) and (4.3), to compute

$$
\begin{aligned}
\left(\nabla \psi_{3}\right)(X, Y) & =-\left(\nabla \psi_{2} \psi_{1}\right)(X, Y)=-\nabla_{X} \psi_{2} \psi_{1} Y+\psi_{2} \psi_{1} \nabla_{X} Y \\
& =-\left[\left(\nabla \psi_{2}\right)\left(X, \psi_{1} Y\right)+\psi_{2}\left(\nabla \psi_{1}\right)(X, Y)+\psi_{2} \psi_{1} \nabla_{X} Y\right]+\psi_{2} \psi_{1} \nabla_{X} Y \\
& =g\left(X, \psi_{1} Y\right)-\psi_{2}\left(\nabla \psi_{1}\right)(X, Y)
\end{aligned}
$$

which in view of equation (4.1), gives

$$
\left(\nabla \psi_{3}\right)(X, Y)+\left(\nabla \psi_{3}\right)(Y, X)=0, \quad X, Y \in \mathfrak{X}\left(S^{5}\right) .
$$

Hence, $\left(\psi_{3}, \xi, \eta, g\right)$ is a nearly cosymplectic structure on $S^{5}$.

\section{References}

[1] Besse, A. L., Einstein Manifolds, Springer Verlag, (1987).

[2] Blair, D. E., Contact manifolds in Riemannian Geometry, Lecture Notes in Math. 509, Springer Verlag (1976).

[3] Blair, D. E, Showers, D. K. and Yano, K., Nearly Sasakian Structures, Kodi Math. Sem. Rep. 27(1976), 175-180.

[4] Ejiri, N. Totally real submanifolds in a 6-sphere, Proc. Amer. Math. Soc. 83 (1981), 759-763.

[5] Okumura, Masafumi. Certain almost contact hypersurfaces in Kaehlerian manifolds of constant holomorphic sectional curvatures. Tôhoku Math. J. (2) 16 (1964), 270-284.

[6] Olszak, Z, Nearly Sasakian manifolds, Tensor (N.S.) 33 (1979), no. 3, 277-286.

[7] Olszak, Z, Five-dimensional nearly Sasakian manifolds, Tensor (N.S.) 34 (1980), no. 3, $273-276$.

\section{Affiliations}

SHARIEF DESHMUKH

AdDRESS: Department of Mathematics, College of Science, King Saud University P.O. Box-2455 Riyadh11451, Saudi Arabia.

E-MAIL: shariefd@ksu.edu.sa

\section{ORCID ID : orcid.org/0000-0003-3700-8164}

IBRAHIM AL-DAYEL

ADDRESS: Department of Mathematics, College of Science, Al-Imam Muhammad Ibn Saud Islamic University P.O. Box-65892, Riyadh-11566, Saudi Arabia.

E-MAIL: ibraheem96@live.com

ORCID ID : orcid.org/0000-0002-5901-2511 